\title{
How Does Autoimmunity Cause Tumor Regression? A Potential Mechanism Involving Cross-Reaction Through Epitope Mimicry
}

\author{
Mouldy Sioud \\ The Norwegian Radium Hospital, Institute for Cancer Research, Department of Immunology, Molecular \\ Medicine Group, Oslo, Norway. \\ Accepted March 21, 2002
}

\begin{abstract}
Background: Although the exact mechanisms mediating the initiation of autoimmune diseases are unknown, sequence similarity between infectious agents and self-proteins (epitope mimicry) has been proposed as the main trigger mechanism. Interestingly, this mechanism of epitope mimicry may also evoke potent tumor immunity. Indeed, experimental data support a beneficial role of autoimmunity in some patients with cancer. Additionally,
\end{abstract}

autoimmunity induced via vaccination with xenogeneic antigens was found to be effective. Thus, the ability to manipulate the immune system via immunologic crossreactions should have important potential in both preventive and therapeutic strategies for cancer. This strategy may break down the friendly established relationship between tumor tissues and the cells of the immune system.

\section{Introduction}

Normally, a functional immune system is able to react against foreign antigens while remaining unresponsive to self-antigens. This self-tolerance is acquired and maintained by combination of central and peripheral tolerances (1). In addition to clonal selection, immune specificity is regulated by receptor selection in $\mathrm{T}$ and $\mathrm{B}$ lymphocytes at different stages of their differentiation (1). Through various specific processes such as positive and negative selections, a peripheral repertoire that is depleted from autoreactive lymphocytes is generated $(1,2)$. To establish unresponsiveness to self during positive selection, the selected thymocytes are expected to increase their activation threshold to self peptides, thereby peripheral $\mathrm{T}$ cells are properly activated by only foreign high affinity peptides. These cells can be regarded as high affinity $\mathrm{T}$ cells (Fig. 1). However, despite the selective pressures imposed on B and $\mathrm{T}$ cells, self-reactive lymphocytes can be found in the blood of healthy individuals $(3,4)$. These findings imply that central and peripheral tolerances are somehow defective. The activation of these potential

Correspondence and reprint requests should be addressed to: M. Sioud, The Norwegian Radium Hospital, Institute for Cancer Research, Department of Immunology, Molecular Medicine Group, Montebello, N-0310 Oslo, Norway. Fax: 4722500 730; e-mail: mosioud@ulrik.uio.no autoreactive lymphocytes is expected to be controlled by various mechanisms of antigen-induced tolerance such as apoptosis, anergy, $\mathrm{T}$ regulatory cells $\left(T_{R}\right)$, and immune deviation $(4,5)$. In autoimmune diseases, self-reactive $\mathrm{T}$ and $\mathrm{B}$ cells become aggressive and cause tissue injury, in some cases leading to severe injuries. How the mechanisms of tolerance mentioned are dysregulated in patients with autoimmune diseases is not well understood.

In principle, the diversity and longevity of peripheral $\mathrm{T}$ and $\mathrm{B}$ cells determine our capacity to mount protective immune responses. However, the realization that the immune response to tumor antigens is unable to eradicate tumor growth has expanded the complexity of molecular interactions between tumor cells and the immune system (6). Our current study, which examines the nature of the immune responses in patients with breast cancer, has indicated that in the long-time survival patients an anti-tumor autoimmunity has occurred and that epitope mimicry could be the initiating mechanism (7-9). Notably, epitope mimicry has been proposed as a mechanism for the induction of autoimmunity. This cross-reaction is expected to tip the balance of immunologic response versus tolerance toward immune response $(10,11)$. This exogenous foreign alarm signal may explain why tumors are sometimes spontaneously rejected. Here, some important aspects of this novel anti-cancer approach are reviewed. 


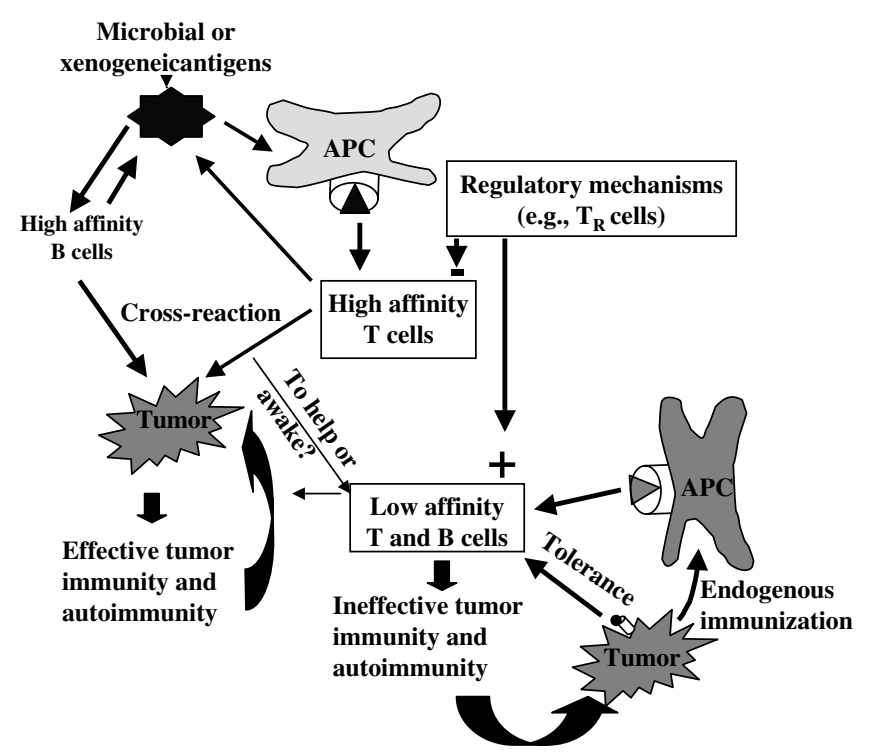

Fig. 1. Epitope mimicry can evoke an effective anti-tumor autoimmunity. The activation of high-affinity $\mathrm{T}$ - and B-lymphocyte precursors with microbial or xenogeneic antigens may cross react with host tissue constituents and eventually break down self-tolerance to tumor antigens. In contrast, the activation of low-affinity T- and B-lymphocyte precursors by tumor antigens may not be sufficient to mount a protective immunity. In addition, tumors can induce tolerance against neoantigens acquired during transformation. (for more details, see the text).

\section{Epitope Mimicry and Autoimmunity}

How autoreactive lymphocytes are regulated in healthy individuals and the factors that affect their dysregulation in autoimmunity is the subject of a number of studies (12-14). An important question is how autoimmunization against, for example, synovial membrane, nucleosomes, and myelin basic protein occurs in rheumatoid arthritis, systemic lupus erythematosus, and multiple sclerosis patients, respectively. The fact that these autoantigens are ubiquitous and present in normal individuals raises the question of why autoimmunity does not happen more often. Fundamental defects in the mechanisms that maintain immunologic tolerance to selfconstituents and environmental factors must play important roles $(5,13)$. However, despite extensive research in this field, the primary triggers that evoke the autoimmune reaction are yet to be identified.

Recently, the concept of epitope mimicry as a mechanism for triggering autoimmunity has received a great deal of attention $(10,11)$. The theory is that an infectious agent (e.g., parasite, bacteria, and virus) displays epitopes immunologically resembling host determinant and due to the mirror antigen differences between the two, the pathogen's epitope subsequently induces an immune response that eventually breaks tolerance to the host epitope. Once the immune system becomes primed to attack the invader, it might eventually destroy normal tissues. For example, bacterial urinary tract infections have been suggested to induce cross-reactive immune responses to antigens in the liver epithelium that contribute to the development of primary biliary cirrhosis.Viral infection has been implicated in multiple sclerosis, and microbial heat shock proteins have been implicated in rheumatoid arthritis (15-18).

The capacity of an epitope mimic to induce an autoimmune disease depends on its appropriate presentation by the host antigen presenting cells, thereby supporting the association between major histocompatibility complex (MHC) products and autoimmune diseases (19). Indeed, the binding association between a given MHC molecule and its peptide, whether it be a self- or foreign peptide involved in either a class I or II interaction, is a genetically controlled event. Because of unique MHC polymorphisms within a population, peptides exhibiting binding capacity in a given host may be completely nonreactive in other individuals of a particular species who lack those MHC alleles (19). This explains in part why autoimmunity does not happen often. However, some autoimmune diseases such as rheumatoid arthritis can develop in the absence of the disease-associated haplotype (20).

Most of the described mimic epitopes (mimotopes) induce autoimmune disease in animal models $(21,22)$. From these studies, it appears that the mechanism(s) by which epitope mimics induce a destructive proliferative rather than anergizing response is (are) different compared to those initiated by direct immunization by self-antigens, where anergy is the main primary outcome. A phenomenon that is generally termed "epitope spreading" has been observed in most animal models for autoimmunity, such as that for experimental autoimmune encephalomyelitis $(22,23)$. Although this phenomenon of epitope spreading is more difficult to study in humans, numerous studies have suggested a diversification of T- and B-cell specificities in human autoimmune diseases (24-28). Epitope spreading may occur either within a single antigen, different antigens from the same tissue structure, or within different antigens that are not physically linked. In this context, and in contrast to patients with autoimmune diseases, cancer patients may immunologically benefit from the phenomenon of epitope spreading, which is more likely to depend on many factors, including the nature of the antigen(s) and the level of established immunologic tolerance.

\section{Cancer and the Immune System}

Cancer development is frequently accompanied by immune response against self- and altered antigens expressed by tumor cells (6). In this regard, autoantibodies to various self-proteins have been found in the sera of patients with solid tumors or hematologic malignancies. This emphasizes the idea that cancer patients can mount tumor immunity, which could be, in part, autoimmunity. In contrast to patients 
with autoimmune diseases, in the majority, if not all, cancer patients the immune system is unable to combat tumor growth. Thus, what are the major difference between immune responses in patients with cancer and autoimmune diseases? Notably, tumors seem to find ways to generate tolerance in the immune system such as the down-regulation of MHC class I molecules and cellular constituents involved in the antigen processing and presentation pathways (29). Tumors can also induce several different biochemical defects in T lymphocytes (30). In addition, the immune response against tumors is hindered by the functional hierarchy in the immunogenicity of T- and B-cell determinants.

$\mathrm{T}$ - and B-cell determinants of protein antigens were divided into dominant, subdominant, and cryptic to reflect their different degree of immunogenicity in vivo (31). T cells reactive with dominant determinants of tumor antigens and perhaps subdominant epitopes are deleted in the thymus during negative selection. Thus, most of the tumor determinants are expected to be immunologically silent; hence an effective tumor immunity can not be induced via self-vaccination. Additionally, as tumor accumulate neoantigens during transformation they also gradually induce tolerance in $\mathrm{T}$ cells against these neoantigens.

Despite these escape mechanisms and the functional properties of the immune system, in few cancer patients spontaneous regression of malignant tumors was observed $(32,33)$. In addition, the presence of some autoantibodies correlated with patient survival. For example, breast cancer patients with a natural humoral response to MUCI and/or heat shock proteins hsp90 exhibited a better outcome $(34,35)$. These clinical observations indicate that in some cancer patients, protective, albeit not strong, immunity and autoimmunity can be mounted. Thus, understanding the nature of the immune response in these patients would facilitate the design of effective cancer vaccines. How does the immune system eliminate tumors in these patients?

Unfortunately, the study of tumor immunity requires prior knowledge of the antigen (protein) sequences. As a consequence of this major limitation, only a relatively small number of antigens have been studied. However, the ability to profile the immune responses with phage-display technology does not require prior structural knowledge of the antigens. Indeed, epitope libraries can identify the specificities of antibodies produced in vivo whether or not the parental antigens are known. The only prerequisite of this novel approach is the availability of patient sera $(36,37)$.

During our studies of the immune responses in patients with breast cancer using phage-display technology, we noted that a subset of patients developed tumor autoimmunity (7-9). More important, patients with high titers of immunoglobulin $\mathrm{G}$ (IgG) antibodies against a 66-kDa and Sp100 autoantigens exhibited an improved outcome. Additionally, patients with the most improved outcome have developed IgG antibodies against most of the selected B-cell peptide epitopes. This observation is in accordance with the phenomenon of epitope spreading seen in patients with autoimmune diseases (22). This is a form of intramolecular and/or intermolecular amplification cascade in which determinants that behave cryptically following primary immunization become immunogenic as the disease progresses. Similar to patients with autoimmune diseases, our patients exhibited mainly a Th-1-type response. Furthermore, the described IgG autoantibodies are not pathogenic; no additional clinical features were seen in our patients than the cancer. Therefore, autoimmunity did not develope into autoimmune disease. From an immunologic standpoint, both B- and T-cell responses may help to produce anti-tumor response in the long-time survival patients. When associated with appropriate alarms, tumors may become seen by the immune system as dangerous invaders. Thus, learning how tolerance is broken in these patients may offer novel strategies to make cancer vaccines. Although $\mathrm{B}$ and $\mathrm{T}$ cells respond to antigens with high specificity, the type of the immune response is expected to be determined by the nature of the antigen, antigen presenting cells (e.g., dendritic cells) and the tissue in which the primary response occurs (e.g., tumors). Understanding the cellular interactions between these players will help us to predict how and when protective immune response against tumors are more likely to be generated.

The potential coupling of tumor immunity with autoimmunity has also been suggested by the clinical observation that patients with metastatic melanoma who develop vitiligo have a better prognosis (38). Notably, there are observations that support a possible protective role for autoimmune diseases in cancer patients. In this respect, the mortality rate of cancer patients with multiple sclerosis was found to be significantly lower than that of cancer patients in general (32).

\section{Epitope Mimicry and Anti-Tumor Immunity}

Most of the IgG antibody specificities that we have identified share a significant homology with human and microbial proteins, thus bringing into question a role for molecular mimicry as the initiating mechanism of tumor autoimmunity seen in our patients (7). In connection with this, long-term remission of malignant brain tumors after intracranial infection has been reported in four patients (39). Additionally, improved survival rates have been reported for cancer patients with microbial infection $(40,41)$.

If the tumor autoimmunity seen in our patients was initiated by an epitope mimicry mechanism, an intriguing question is whether this type of autoimmunity is beneficial in the treatment of tumors in vivo. 
Notably, a significant homology at protein level was found between human proteins and proteins from other species. Thus, this sequence similarity can be used as trigger to breakdown immunologic tolerance to self-proteins, especially those expressed by tumors.

As a first step, we investigated the feasibility of breaking immune tolerance in inbred syngeneic rats against self-malignant gliomas via vaccination with their human counterparts. Immunization of rats with human glioma proteins inhibited tumor growth, whereas no significant anti-tumor effect was obtained when rat glioma proteins were used as an immunogen (M. Sioud and D. Sørensen, unpublished results). Notably, the immunogenicity of rat glioma proteins was very poor. In contrast, their human counterparts induced a IgG immune response that cross reacted with rat glioma proteins. Thus, self-tolerance against tumors can be broken via self-foreign antigen crossreactivity.

Although the exact roles of antibodies on tumor growth remains to be investigated, antibodies might either mediate antibody-dependent cellular cytotoxicity, induce complement-mediated lysis, or in some cases trigger apoptotic cell death (42). The described antibodies are IgG, which predicts the coexistence of helper $\mathrm{T}$ cells. In accordance with our observations, a recent study demonstrated that immune tolerance against autologous angiogenic endothelial cells can be broken by xenogeneic antigen from endothelial cells (43). Interestingly, the Ig were found to be effective in blocking endothelial cell proliferation. Furthermore, the anti-tumor effect was found to be $\mathrm{CD} 4^{+}$T-cell-dependent.

\section{Conclusion}

Because tumors raise from own host tissues, appropriate (e.g., high-affinity) $\mathrm{T}$ and $\mathrm{B}$ cells specific for tumor antigens are expected to be deleted from the periphery. Thus, self-immunization by tumor antigens may prime only a pool of precursor lymphocytes with low avidity for cryptic determinants. In addition, the activity of these lymphocytes could be held under the control of regulatory mechanisms such anergy and interaction with $\mathrm{T}$ regulatory cells. Consequently, the mounted immunity and autoimmunity may not be sufficient to reject cancers (Fig. 1). In contrast, exogenous immunization via epitope mimicry may prime a second pool of precursor lymphocytes exhibiting high affinity toward the xenogeneic dominant determinants and having the potential to cross react with dominant, subdominant, or cryptic tumor determinants. This pool of precursor lymphocytes is expected to escape the control pressure imposed by the regulatory mechanisms mentioned because since they have been positively selected to mount effective immunity against foreigner invaders. Furthermore, T-T and/or T-B cell cooperation mediated by immunologic cross-reactions may render immunologically silent tumor determinants (cryptic epitopes) immunogenic. Notably, determinants presented cryptically during the establishment of tolerance in the thymus and the secondary lymphoid tissues may be displayed dominantly under certain conditions (31). Factors affecting the display of a determinant as dominant or cryptic include antigen processing, lymphocyte competition for antigenbearing APC, as well as the intracellular versus extracellular origin of the autoantigens $(31,44,45)$.

In light of these potential cellular interactions, the immune system may respond to the tumors as if they are foreign and mount an effective tumor immunity and autoimmunity (Fig. 1). These observations, together with the fact that self-tolerance can be overcome with the use of mimic antigens, should facilitate the design of cancer vaccines, as exemplified by the recent human clinical trial with the mouse prostatic acid phosphatase (46). However, we need to find the balance between sufficient autoimmunity to inhibit tumor growth, while avoiding detrimental autoimmune attack of normal tissues (47). Thus, appropriate mimic antigens must be characterized.

\section{Acknowledgments}

Our research was supported by grants from the Norwegian Cancer Society and the Norwegian Foundation for Health and Rehabilitation. I thank Drs. Øyvind Melien and Mona H. Hansen for critical reading of the manuscript and all the members of the group who contributed to the work discussed here.

\section{References}

1. Parkin J, Cohen B. (2001) An overview of the immune system. Lancet 357: 1777-1789.

2. Nemazee D. (2000) Receptor selection in B and T lymphocytes. Annu. Rev. Immunol. 18: 19-51.

3. Burns J, Rosenzweig A, Zweiman B, Lisak RP. (1983) Isolation of myelin basic protein-reactive $\mathrm{T}$ cell lines from normal human blood. Cell Immunol. 81: 435-440.

4. Arnold B, Schonrich G, Harmmerling GJ. (1993) Multiple levels of peripheral tolerance. Immunol. Today 14: 12-14

5. Maloy KJ, Powrie F. (2001) Regulatory T cells in the control of immune pathology. Nat. Immunol. 2: 816-822.

6. Jäger D, Jäger E, Knuth A. (2001) Immune responses to tumor antigens: implications for antigen specific immunotherapy of cancer. J. Clin. Pathol. 54: 669-674.

7. Hansen MH, Østenstad B, Sioud M. (2001) Antigen-specific IgG antibodies in stage IV long-term survival breast cancer patients. Mol. Med. 7: 230-239.

8. Hansen MH, Østenstad B, Sioud M. (2001) Identification of immunogenic antigens using a phage-displayed cDNA library from an invasive ductal breast carcinoma tumour. Int. J. Oncol. 19: 1303-1309.

9. Sioud M, Hansen MH. (2001) Profiling the immune response in patients with breast cancer by phage-displayed cDNA libraries. Eur. J. Immunol. 31: 716-725.

10. Benoist C, Mathis D. (2001) Autoimmunity provoked by infection: how good is the case for T cell epitope mimicry? Nat. Immunol. 2: 797-801.

11. Davis JM. (1997) Molecular mimicry: can epitope mimicry induce autoimmune disease? Immunol. Cell Biol. 75: 113-126.

12. Mocci S, Lafferty K, Howard M. (2000) The role of autoantigen in autoimmune disease. Curr. Opin. Immunol. 12: 725-730. 
13. Maverakis E, van den Elzen P, Sercarz E. (2001) Self-reactive $\mathrm{T}$ cells and degeneracy of $\mathrm{T}$ cell recognition: evolving concepts-from sequence homology to shape mimicry and TCR flexibility. J. Autoimmun. 16: 201-209.

14. Steinman L. (1996) A few autoreactive cells in an autoimmune infiltrate control a vast population of nonspecific cells: A tale of smart bombs and the infantry. Proc. Natl. Acad. Sci. U.S.A. 93: 2253-2256.

15. Fujinami RS. (2001) Can virus infections trigger autoimmune disease? J. Autoimmun. 16: 229-234.

16. Theil DJ, Tsunoda I, Rodriguez F, Whitton JL, Fujinami RS. (2001) Viruses can silently prime for and trigger central nervous system autoimmune diseases. J. Neurovirol. 7: 220-227.

17. Todryk SM, Melcher AA, Dalgleish AG, Vile RG. (2000) Heat shock proteins refine the danger theory. Immunology 99: 334-337.

18. Quale AJ, Wilson KB, Li SG, et al. (1992) Peptide recognition, $\mathrm{T}$ cell receptor usage and HLA restriction elements of human heat-shock protein (hsp) 60 and mycobacterial 65-kDa hsp-reactive $\mathrm{T}$ cell clones from rheumatoid synovial fluid. Eur. J. Immunol. 22: 1315-1322.

19. McDevitt HO. (1998) The role of MHC class II molecules in susceptibility and resistance to autoimmunity. Curr. Opin. Immunol. 10: $677-681$

20. Wandstrat A, Wakeland E. (2001) The genetic of complex autoimmune diseases: non-MHC susceptibility genes. Nat. Immunol. 2: 802-909.

21. Myers LK, Rosloniec EF, Cremer MA, Kang AH. (1997) Collagen-induced arthritis, an animal model for autoimmunity. Life Sci. 61: 1861-1878.

22. Gordon TP. (1998) Determinant spreading: lessons from animal models and human disease. Immunol. Rev. 164: 209-229.

23. Lehmann PV, Forsthuber T, Miller A, Sercarz EE(1992) Spreading of T-cell autoimmunity to cryptic determinants of an autoantigen. Nature 359: 155-157.

24. Lipsky PE, Dorner T. (2001) Immunoglobulin variable-region gene usage in systemic autoimmune diseases. Arthritis Rheum. 44: $2715-2727$.

25. Gold DP. (1994) TCR V gene usage in autoimmunity. Curr. Opin. Immunol. 6: 907-912.

26. Sioud M, Kjeldsen-Kragh J, Suleyman S, Vinje O, Natvig JB, Forre Ø. (1992) Limited heterogeneity of T cell receptor variable region gene usage in juvenile rheumatoid arthritis synovial T cells. Eur. J. Immunol. 22: 2413-2418.

27. Dybwad A, Førre Ø, Natvig JB, Sioud M. (1995) Structural characterization of peptides that bind synovial fluid antibodies from patients: a novel strategy for identification of disease-related epitopes using a random peptide library. Clin. Immunol. Immunopathol. 75: 45-50.

28. Potter KN, Thomson RK, Hamblin A, Richards SD, Lindsay JG, Stevensen FK. (2001) Immunogenetic analysis reveals that epitope shifting occurs during B-cell affinity maturation in primary biliary cirrhosis. J. Mol. Biol. 306: 37-46.

29. Salih HR, Nussler V. (2001) Immune escape versus tumour tolerance: how do tumours evade immune surveillance? Eur. J. Med. Res. 27: 323-32.

30. Stavely-O'Carrol K, Sotomayor E, Montgomery J, et al. (1998) Induction of antigen-specific $T$ cell anergy: an early event in the course of tumour progression. Proc. Natl. Acad. Sci. U.S.A. 95: 1178-1183.
31. Sercarz EE, Lehmann PV, Ametani A, Benichou G, Miller A, Moudgil K. (1993) Dominance and crypticity of T cell antigenic determinants. Annu. Rev. Immunol. 11: 729-766.

32. Palo J, Duchesne J, Wikstrom J. (1977) Malignant diseases among patients with multiple sclerosis. J. Neurol. 216: 217222.

33. Paul R, Remes K, Lakkala T, Pelliniemi TT. (1994) Spontaneous remission in acute myeloid leukaemia. Br. J. Haematol. 86: $210-212$.

34. von Mensdorff-Pouilly S, Gourevitch MM, Kenemans P, et al. (1996) Humoral immune response to polymorphic epithelial mucin 1 in patients with benign and malignant breast tumours. Eur. J. Cancer 32: 1325-1331.

35. Conroy SE, Latchman DS. (1996) Do heat shock proteins have a role in breast cancer? Br. J. Cancer 74: 717-721.

36. Dybwad A, Forre Ø, Kjeldsen-Kragh J, Natvig JB, Sioud M. (1993) Identification of new B cell epitopes in the sera of rheumatoid arthritis using a random nanopeptide phage library. Eur. J. Immunol. 23: 3189-3193.

37. Sioud M, Førre Ø, Dybwad A. (1996) Selection of ligands for polyclonal antibodies from random peptide libraries: potential identification of (auto)antigens that may trigger B and T cell responses in autoimmune diseases. Clin. Immunol. Immunopathol. 79: 105-114

38. Bystryn JC, Rigel D, Friedman RJ, Kopf A. (1984) Prognostic significance of hypopigmentation in malignant melanoma. Arch. Dermatol. 123: 1053-1055.

39. Bowles AP, Perkins E. (1999) Long-term remission of malignant brain tumors after intracranial infection: a report of four cases. Neurosurgery 44: 636-643.

40. Papachristou DN, Forster JG. (1979) Effect of postoperative wound infection on the course of stage II melanoma. Cancer 43: 1106-1111.

41. Pizzo PA, Commers J, Cotton D, et al. (1984) Approaching the controversies in anti-bacterial management of cancer patients. Am. J. Med. 76: 436-448.

42. Kummer U, Staerz US. (1993) Concepts of antibody-mediated cancer therapy. Cancer Invest. 11: 174-188.

43. Wei Y, Huang MJ, Yang L, et al. (2001) Immunogene therapy of tumors with vaccine based on Xenopus homologous vascular endothelial growth factor as a model antigen. Proc. Natl. Acad. Sci. U.S.A. 98: 11545-11550.

44. Kedl RM, Rees WA, Hildeman DA, et al. (2000) T cells compete for access to antigen-bearing antigen-presenting cells. J. Exp. Med. 192: 1105-1113.

45. Pion S, Christianson GJ, Fontaine P, Roopenian DC, Perreault C. (1999) Shaping the repertiore of cytotoxic T lymohocyte response: explanation for the immunodominance effect whereby cytotoxic $\mathrm{T}$ lymphocytes specific for immunodominant antigens prevent recognition of nondominant antigens. Blood 93: 952-962.

46. Fong L, Brockstedt D, Benike C, et al. (2001) Dendritic cell-based xenoantigen vaccination for prostate cancer immunotherapy. J. Immunol. 167: 7150-7156.

47. Naftzger C, Takechi Y, Kohda H, Hara I, Vijayasaradhi, Houghton AN. (1996) Immune response to a differentiation antigen induced by altered antigen: A study of tumor rejection and autoimmunity. Proc. Natl. Acad. Sci. U.S.A. 93: 14809-14814. 